\title{
Optimization of the encapsulation efficiency of a novel oral insulin delivery nanosystem
}

\author{
Optimização da eficiência de encapsulaçãode um novo nanosistema para a administração oral \\ de insulina
}

\author{
Carolina Correia ${ }^{1}$, Patrícia Rijo ${ }^{2,3}$, Lia Ascensão ${ }^{4}$, Marisa Nicolai ${ }^{2}$, Diogo Matias $^{2}$, Catarina Pinto Reis ${ }^{2}$ \\ ${ }^{1}$ Universidade Lusófona de Humanidades e Tecnologias, Campo Grande, 376, 1749-024 Lisboa \\ ${ }^{2}$ CBIOS - Universidade Lusófona's Research Center for Biosciences and Health Technologies (UDE), Campo Grande 376, \\ 1749-024, Lisboa, Portugal \\ ${ }_{3}^{3}$ iMed.UL - Research Institute for Medicines and Pharmaceutical Sciences, Faculdade de Farmácia da Universidade de \\ Lisboa, Lisboa, Portugal \\ ${ }^{4}$ IBB - Centro de Biologia Vegetal, Universidade de Lisboa, Faculdade de Ciências, Departamento de Biologia Vegetal, \\ Lisboa, Portugal \\ Email: catarina.reis@ulusofona.pt
}

\begin{abstract}
Oral delivery of insulin may significantly improve the quality of life of diabetes patients who routinely receive insulin by the subcutaneous route. The oral delivery of insulin remains a challenge because of its limited absorption. The main goal of this study was to produce stable nanoparticles with high loads of insulin for further in vivo testing. Poli(lactic-co-glycolic acid) (PLGA), a biodegradable and biocompatible polymer, revealed to be a good presupposition for this approach. Nanoparticles were produced through a multiple emulsion method and the encapsulation efficiency was accessed by a HPLC method. Monodispersed nanoparticles were produced and the insulin EE were improved from $30 \%$ to $65 \%$, controlling parameters such as the stirring speed rate, volume of external phase, $\mathrm{pH}$ range, type of insulin, type of surfactant and polymer concentrations. The nanoparticle size was $398.6 \mathrm{~nm}$ with a polydispersivity index of 0.19 . The presence of a salt concentration between $20 \mathrm{~g}$ L-1 and $50 \mathrm{~g} \mathrm{~L}-1$ and the type of insulin decreased the size of nanoparticles. Bioactivity assessment after the encapsulation process and full characterization of physical-chemical interactions between all species is now required.
\end{abstract}

Keywords: PLGA, oral insulin nanosystem, multiple emulsion method, encapsulation efficiency

\section{Resumo}

A administração oral de insulina pode melhorar significativamente a qualidade de vida dos doentes diabéticos que administram de forma crónica insulina por via subcutânea. Porém, a administração oral de insulina continua a ser um desafio devido à absorção limitada. Este estudo teve como objectivo a produção de nanopartículas estáveis e com capacidade de encapsulação da insulina para futuros testes in vivo. O ácido poli(láctico-co-glicólico (PLGA), um polímero biodegradável e biocompatível, revelou ser um bom candidato para este tipo de abordagem. As nanopartículas foram produzidas por um método de emulsão múltipla e a eficiência de encapsulação foi determinada por HPLC. Produziram-se nanopartículas monodispersas e melhorouse a eficiência de encapsulação de $30 \%$ para $65 \%$, controlando os diferentes parâmetros tais como a velocidade de agitação, o volume da fase externa, o intervalo de $\mathrm{pH}$, o tipo de insulina e as concentrações de polímero. As nanopartículas apresentaram um tamanho de 398,6 nm e um índice de polidispersividade de 0,19. A presença de uma concentração de sal entre $20 \mathrm{~g} \mathrm{~L}-1$ e 50 g L-1 e o tipo de insulina utilizado diminuíram o tamanho das nanopartículas. São agora necessários ensaios de bioatividade após o processo de encapsulação e a caracterização total das interações físicas e químicas entre todas as espécies envolvidas.

Palavras-chave: PLGA, nanossistema de insulina oral, emulsão múltipla, eficiência de encapsulação 


\section{Introduction}

Insulin, a polypeptide secreted by the $\beta$ cells of the pancreatic islets of Langerhans, is a critical hormone to metabolic regulation [1]. The molecular weight (MW) of insulin is around $6 \mathrm{kDa}$ with an isoelectric point of 5.3 [2]. Disorders regarding insulin secretion and their action mechanism leads to the onset of Diabetes Mellitus, a prevalent condition with serious risks to human health [3]. Clinically Diabetes Mellitus is divided into four classes: type 1, which is derived from a destruction of $\beta$ cells, leading to a completely deficient secretion of insulin; type 2, in which insulin secretion is gradually affected due to a background of resistance mechanisms to its action; the gestational one, which occurs during the pregnancy and the other one due to genetic causes, pancreatic disease, or even due to a chemical or drug action [4]. In this context, the presence of adequate plasmatic levels of insulin emerges as a fundamental tool for metabolic control.

Currently, diabetes type 1 treatment involves a chronic and daily subcutaneous administration of insulin, which generates not only a low patient compliance, as well as a local lipodystrophies and a low liver control with an inherent high risk of hypoglycemic episodes [5]. Although their use is essential in type 1 , the introduction of insulin in the remaining clinical forms may also be required in combination with oral anti diabetics [6]. Thus, an alternative and noninvasive way of insulin administration is required. The oral route may represent the answer for this issue but it has been contested mainly because of a low insulin bioavailability. Herein, several points are present such as high instability of proteins in acidic medium, low capacity of intestinal permeation mainly due to its high hydrophilicity and molecular weight (MW) and the rapid enzymatic degradation suffering along the gastrointestinal tract [7-9]. Various systems have been designed such as microemulsions, liposomes and particularly, nanoparticles [10]. This last approach has been giving excellent results, not only because of the high area of contact of the particle surface and intestinal cell, which facilitates the paracellular path and the uptake by the enterocytes and $M$ cells [11], but also by the enzymatic protection and a higher half-life time of drugs in the blood stream [12]. In this field, some factors may be considered during nanoparticle production. In order to improve the time contact between the nanoparticles and the absorption site it i necessary to control the size and the surface charge of the particle. It is also known that mucus is surrounded by strongly anionic groups, then the interaction may be facilitated by the presence of cationic or neutral groups $[5,13]$. Another relevant aspect is the intrinsic toxicity of residual levels of solvents in the formulations. The International Conference of Harmonization (ICH) guidelines may be followed. Biocompatibility and bio-

\section{Introdução}

A insulina, um polipeptídeo secretado pelas células $\beta$ dos ilhéus pancreáticos de Langerhans, é uma hormona essencial para a regulação metabólica [1]. A massa molecular (MM) da insulina é de cerca de $6 \mathrm{kDa}$ com um ponto isoeléctrico de 5,3 [2]. Perturbações a nível da secreção de insulina e do seu mecanismo de acção levam ao aparecimento da Diabetes Mellitus, uma doença prevalente e com sérios riscos para a saúde humana [3]. Clinicamente consideram-se quatro tipos de Diabetes Mellitus:, o tipo 1, que resulta da destruição das células $\beta$, originando uma total deficiência na produção de insulina; o tipo 2 , em que progressivamente a secreção de insulina é afectada devido a mecanismos antecedentes de resistência à sua acção; a gestacional, que ocorre durante a gravidez, e outra que tem como origem causas de ordem genética, doença pancreática, substâncias químicas ou farmacológicas [4]. Neste contexto, a presença de níveis plasmáticos adequados de insulina surgem como uma ferramenta fundamental para a regulação metabólica. Atualmente, o tratamento da diabetes tipo 1 envolve uma administração subcutânea, crónica e diária de insulina, o que gera não só uma menor adesão por parte do doente, como outros efeitos, tais como lipodistrofias locais e um baixo controlo hepático, inerente a um alto risco de hipoglicémia [5]. Embora a utilização de insulina seja fundamental na terapia da Diabetes Mellitus do tipo 1, nos restantes tipos a sua utilização poderá ser igualmente necessária em combinação com os antidiabéticos orais [6]. Assim, é necessário encontrar uma forma alternativa e não invasiva de administração de insulina. A via oral, que pode ser a resposta a esta questão, tem sido contestada principalmente pela baixa biodisponibilidade da insulina. Facto para o qual concorrem vários factores, tais como a elevada instabilidade das proteínas em meio ácido, a baixa capacidade de permeação intestinal, principalmente devido à sua elevada hidrofilia, massa molecular (MM) e rápida degradação enzimática ao longo do trato gastrointestinal (GI) [7-9].

Para ultrapassar estes problemas, vários sistemas têm sido concebidos, como microemulsões, lipossomas e, em particular, nanopartículas [10]. Esta última abordagem tem vindo a dar excelentes resultados, não só devido à elevada área de contacto entre a superfície da partícula e a célula intestinal, o que facilita a sua passagem paracelular e a captação pelos enterócitos e células M [11], mas também pela protecção enzimática e aumento do tempo de semi-vida dos fármacos na corrente sanguínea [12]. Neste campo, alguns factores podem ser considerados durante a produção das nanopartículas. Para melhorar o tempo de contacto entre as nanopartículas e o local de absorção é necessário o controlo do tamanho e da carga da superfície da partícula. Uma vez que o muco é rodeado por grupos fortemente aniónicos, a interacção com as nanopartículas pode ser facilitada pela presença de grupos catiónicos ou neutros $[5,13]$. Um outro aspecto importante é a toxicidade intrínseca dos níveis residuais de solventes presentes nas formulações. As orientações da Conferência Internacional de Harmonização (ICH) 
degradability of the encapsulant material may also be considered.

Poly(lactic-co-glycolic) acid (PLGA) appears as the polymer chosen and has been recognized by the Food and Drug Administration (FDA) as safe [14,15]. After administration, PLGA undergoes a hydrolysis reaction generating the original monomers, the glycolic acid and the lactic acid, compounds that also play an important role in human physiological metabolism [16]. Actually, several drugs have already been encapsulated into PLGA nanoparticles. Table 1 shows some of these examples.

One problem that may arise from PLGA encapsulation is the low encapsulation efficiency of insulin. However different strategies have been used to improve this parameter in a more manageable way. Table 2 describes some examples.

In this study, several parameters were tested in order to improve the quantity of insulin encapsulated into PLGA nanoparticles produced by a multiple emulsion method. devem ser meticulosamente seguidas. A biocompatibilidade e a biodegradabilidade do material encapsulante é igualmente um factor importante a considerar.

O ácido poli(láctico-co-glicólico (PLGA) aparece como o polímero escolhido e reconhecido pela Food and Drug Administration (FDA) como seguro $[14,15]$. Após a administração, o PLGA sofre uma reação de hidrólise, gerando os monómeros iniciais, o ácido glicólico e o ácido láctico, compostos que também desempenham um papel importante no metabolismo fisiológico humano [16]. Actualmente, são já vários os fármacos que foram encapsulados em nanopartículas de PLGA. A Tabela 1 mostra alguns desses exemplos.

Um problema que pode surgir com o PLGA é a sua baixa capacidade de encapsulação da insulina. Contudo diferentes estratégias têm sido utilizadas para melhorar este parâmetro de uma forma exequível. A Tabela 2 descreve alguns exemplos.

Neste estudo, foram testados diversos parâmetros para melhorar a quantidade de insulina encapsulada em nanopartículas de PLGA produzidas por um método de emulsão múltipla.

Table 1/ Tabela 1. Type of encapsulated drugs, methods and particle size obtained from the use of PLGA.

Tipos de fármacos encapsulados, métodos e granulometria obtida a partir da utilização do PLGA.

\begin{tabular}{|c|c|c|c|}
\hline $\begin{array}{l}\text { Methods } \\
\text { Métodos }\end{array}$ & $\begin{array}{l}\text { Drug type } \\
\text { Fármaco }\end{array}$ & $\begin{array}{c}\text { Particle size } \\
\text { Tamanho de } \\
\text { partícula, } d / \mathrm{nm}\end{array}$ & $\begin{array}{l}\text { References } \\
\text { Referências }\end{array}$ \\
\hline \multirow{5}{*}{$\begin{array}{l}\text { Solvent evaporation } \\
\text { Evaporação do solvente }\end{array}$} & $\begin{array}{l}\text { Adriamycin } \\
\text { Adriamicina }\end{array}$ & $65-100$ & [17] \\
\hline & $\begin{array}{l}\text { Bufalin } \\
\text { Bufalina }\end{array}$ & 164-184 & {$[18]$} \\
\hline & Cyclosporin A & 300 & [19] \\
\hline & DNA & 100 & [20] \\
\hline & $\begin{array}{l}\text { Doxorrubicin } \\
\text { Doxorrubicina }\end{array}$ & 468 & [21] \\
\hline \multirow{6}{*}{$\frac{\frac{\text { Solvent displacement }}{\text { Deslocamento do }}}{\text { solvente }}$} & $\begin{array}{l}\text { Indometacin } \\
\text { Indometacina }\end{array}$ & 168 & \\
\hline & $\begin{array}{l}\text { Doxorrubicin } \\
\text { Doxorrubicina }\end{array}$ & 274 & \\
\hline & $\begin{array}{l}\text { Valproic acid } \\
\text { Ácido valpróico }\end{array}$ & 166 & \\
\hline & $\begin{array}{l}\text { Phenobarbital } \\
\text { Fenobarbital }\end{array}$ & 157.4 & [22] \\
\hline & $\begin{array}{l}\text { Ketoprofen } \\
\text { Cetoprofeno }\end{array}$ & 67.4 & \\
\hline & $\begin{array}{l}\text { Vancomicin } \\
\text { Vancomicina }\end{array}$ & 186.9 & \\
\hline \multirow{4}{*}{$\begin{array}{l}\frac{\text { Emulsification/Solvent }}{\frac{\text { diffusion }}{\text { Emulsão/Difusão do }}} \\
\underline{\text { solvente }}\end{array}$} & $\begin{array}{c}\text { Tamoxifen citrate } \\
\text { Citrato de tamoxifeno }\end{array}$ & 276 & [23] \\
\hline & $\begin{array}{l}\text { 5-Fluoracile } \\
\text { 5-Fluoracilo }\end{array}$ & $150-190$ & [24] \\
\hline & $\begin{array}{l}\text { Porphyrins } \\
\text { Porfirinas }\end{array}$ & $117-118$ & [25] \\
\hline & $\begin{array}{l}\text { Curcumin } \\
\text { Curcumina }\end{array}$ & 80.9 & [26] \\
\hline $\begin{array}{c}\frac{\text { System of fluid }}{\text { nanoprecipitation }} \\
\underline{\text { Sistema de }} \\
\text { nanoprecipitação fluída }\end{array}$ & - & $140-500$ & [27] \\
\hline $\begin{array}{l}\mathrm{W} / \mathrm{O} / \mathrm{W} \\
\mathrm{A} / \mathrm{O} / \mathrm{A}\end{array}$ & Minocicline & $139-226$ & [28] \\
\hline
\end{tabular}


Table 2/ Tabela 2. Some approaches for optimization of insulin encapsulation efficiency (EE, \%) Algumas estratégias para a optimização da eficiência de encapsulação da insulina (EE, \%).

\begin{tabular}{|c|c|c|c|c|}
\hline $\begin{array}{l}\text { Strategies } \\
\text { Estratégias }\end{array}$ & $\begin{array}{c}\text { Substances added } \\
\text { Substâncias adicionadas }\end{array}$ & EE / \% & $\begin{array}{c}\text { Particle size } \\
\text { Tamanho de } \\
\text { partícula, } d / \mathrm{nm}\end{array}$ & $\begin{array}{l}\text { References } \\
\text { Referências }\end{array}$ \\
\hline \multirow[t]{4}{*}{ HIP } & $\begin{array}{l}\text { Dextran sulfate } \\
\text { Sulfato de Dextrano }\end{array}$ & 79.7 & 201.6 & [29] \\
\hline & $\begin{array}{l}\text { Sodium oleate } \\
\text { Oleato de Sódio }\end{array}$ & 91.2 & 160 & [30] \\
\hline & $\begin{array}{l}\text { Sodium deoxycholate } \\
\text { Deoxicolato de Sódio }\end{array}$ & & & \\
\hline & & 93.6 & 278 & [31] \\
\hline $\begin{array}{l}\text { Reverse micelles } \\
\text { Micelas Reversas }\end{array}$ & $\begin{array}{c}\text { Soya-bean } \\
\text { Phosphatidylcholine } \\
\text { Fosfatidilcolina de soja }\end{array}$ & 90 & 200 & [32] \\
\hline $\begin{array}{l}\text { Ionic gelification } \\
\text { Gelificação Iónica }\end{array}$ & $\begin{array}{l}\text { Alginate } \\
\text { Alginato }\end{array}$ & 80 & 278 & [33] \\
\hline $\begin{array}{l}\text { Nanoprecipitation with an } \\
\text { extra step } \\
\text { Nanoprecipitação com um } \\
\text { passo extra }\end{array}$ & $\begin{array}{l}\text { Solvent: Acetonitrile } \\
\text { Solvente: Acetonitrilo }\end{array}$ & 87 & $300-400$ & [34] \\
\hline $\begin{array}{l}\text { Hydrophobic system } \\
\text { Sistema hidrofóbico }\end{array}$ & $\begin{array}{l}\text { Stearic acid, soya-bean } \\
\text { phosphatidylcholine, } \\
\text { hydroxypropyl- } \beta- \\
\text { cyclodextrin } \\
\text { Ácido esteárico, } \\
\text { Fosfatidilcolina de soja e } \beta \\
\text { hidroxipropil ciclodextrina }\end{array}$ & 95.7 & 526 & [35] \\
\hline $\begin{array}{l}\text { PEGylation } \\
\text { PEGilação }\end{array}$ & PEG & 87 & 260 & [36] \\
\hline $\begin{array}{c}\text { Multiple emulsion W/O/W } \\
\text { Emulsão Múltipla } \\
\text { A/O/A }\end{array}$ & $\begin{array}{l}\text { Solvents: methylene } \\
\text { chloride / Acetone } \\
\text { Solventes: Cloreto de } \\
\text { Metileno/Acetona }\end{array}$ & 94 & 181.90 & [37] \\
\hline
\end{tabular}

\section{Materials and Methods}

\section{Materials}

PURASORB ${ }^{\circledR}$ PLDG 5002-PLGA Ratio L/G\% 50:50 (MW between 45,000 Da and 75,000 Da) was purchased from PURAC (Gorinchem, Netherlands). Pluronic ${ }^{\circledR}$ F68 and Polyvinyl alcohol (PVA) were obtained from Sigma-Aldrich $^{\circledR}$ (Steinheim, Germany). Three different types of insulin were applied: Insulin Novomix ${ }^{\circledR}$ and Novorapid $^{\circledR}$, with a 100 UI mL $^{1}$ concentration, obtained from Novonordisk and Insulin Insuman ${ }^{\circledR}$, with the same concentration, purchased from Sanofi. All chemicals or solvents were of analytical grade.

\section{Preparation and characterization of the nanoparticles}

As summarized in Fig. 1, nanoparticles were produced by a water-in-oil-water $(\mathrm{W} / \mathrm{O} / \mathrm{W})$ multiple emulsion, which is a system with a water reservoir phase inside of oil droplets, surrounded by an external water phase [38].

\section{Materiais e Métodos}

\section{Materiais}

PURASORB $^{\circledR}$ PLDG 5002-PLGA Rácio L/G\% 50:50 (MM entre $45000 \mathrm{Da}$ e $75000 \mathrm{Da}$ ) foi comprado à PURAC (Gorinchem, Holanda). Pluronic ${ }^{\mathbb{R}} \mathrm{F} 68$ e o álcool polivinílico (PVA) foram adquiridos à Sigma-Aldrich ${ }^{\circledR}$ (Steinheim, Alemanha). Foram usados três diferentes tipos de insulina: Insulina Novomix ${ }^{\circledR}$ e Novorapid $^{\circledR}$ com

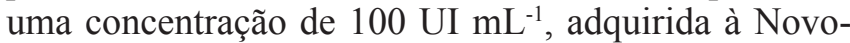
nordisk, e Insulina Insuman ${ }^{\circledR}$ com a mesma concentração, obtida na Sanofi. Todos os produtos químicos e solventes utilizados apresentavam pureza analítica.

\section{Preparação e caracterização das nanopartículas}

Como resumido na Fig. 1, as nanopartículas foram produzidas por uma emulsão múltipla do tipo (A/O/A), que é um sistema com um reservatório aquoso dentro de gotículas de óleo, delimitado por uma fase externa aquosa [38]. Inicialmente o polímero foi dissolvido numa mistura de solventes, acetona/etanol (de respectiva proporção em 


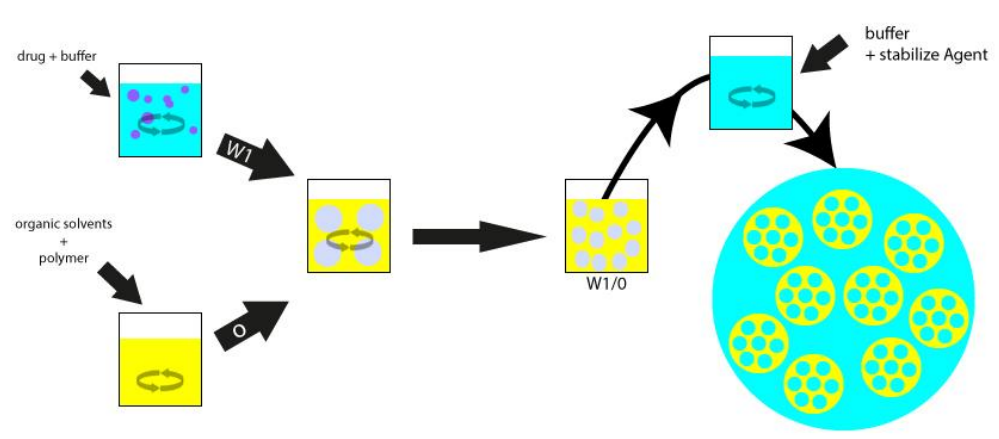

Figure 1/ Fifura 1. Steps of the multiple emulsion method (W/O/W). Etapas do método de emulsão múltipla (A/O/A).

First, the polymer was dissolved in a mixture of acetone/ethanol (with 8:2 respective volume proportions), under a magnetic stirring condition. A buffered solution was added to insulin to adjust the $\mathrm{pH}$ to 4.5 . After the first emulsion was formed, this system was added to the external water phase stabilized with a surfactant agent with or without a salt. Different parameters were accessed such as the stirring speed $(800 \mathrm{rpm}, 1000 \mathrm{rpm}$ and $1400 \mathrm{rpm})$, external phase $(5 \mathrm{~mL}$ or $20 \mathrm{~mL}), \mathrm{pH}$ conditions, type of insulin, type of surfactant (Pluronic ${ }^{\circledR} \mathrm{F} 680.1 \%$ or PVA $2 \%$ ), PLGA mass (75 mg or 100 $\mathrm{mg})$ and the presence or absence of $\mathrm{NaCl}\left(0 \mathrm{~g} \mathrm{~L}^{-1}, 20 \mathrm{~g}\right.$ $\mathrm{L}^{-1}, 50 \mathrm{~g} \mathrm{~L}^{-1}$ or $\left.100 \mathrm{~g} \mathrm{~L}^{-1}\right)$. Nanoparticles were characterized in terms of size and polydispersivity index (PDI) using the Delsa ${ }^{\mathrm{TM}}$ NanoC (Beckman Coulter, USA). Morphology was observed by scanning electron microscopy (SEM). An aliquot of each sample was placed in a glass coverslip and left to dry in a desiccator for SEM analysis. The material was then coated with a thin layer of gold (approximately $500 \mathrm{~nm}$ thick) and observed on a JEOL 5200LV scanning electron microscope (JEOL Ltd., Tokyo, Japan) at an accelerating voltage of $20 \mathrm{kV}$. Images were recorded digitally. Table 3 summarizes the different formulation and process parameters used for preparation of all batches (A - I).

\section{Encapsulation efficiency (EE, \%)}

The percentage of insulin encapsulated into the PLGA nanoparticles was determined in the supernatant (non-encapsulated insulin after centrifugation at 14,000 rpm, during $10 \mathrm{~min}$ ) using a HPLC method under isocratic conditions at a flow rate of $0.8 \mathrm{~mL} \mathrm{~min}{ }^{-1}$ with UV detector at $214 \mathrm{~nm}$ (Column LiChrospher 100, RP-18 (5 $\mu \mathrm{m}, 250 \mathrm{~mm} \times 4 \mathrm{~mm}$ i.d.), Merck (Germany). An aqueous solution of $\mathrm{Na}_{2} \mathrm{SO}_{4}$ anhydrous $(0.2 \mathrm{M})$, adjusted to $\mathrm{pH} 2.3$ with phosphoric acid and acetonitrile (with $73: 27$ respective volume proportions), was used as the volume 8:2), sempre com agitação constante. Posteriormente, a insulina foi adicionada uma solução tamponada de acetato de sódio, para ajustar o $\mathrm{pH}$ a 4,5. Depois da formação da primeira emulsão, o sistema foi adicionado à fase externa aquosa, estabilizada por um agente surfactante, com ou sem a presença de um sal. Diferentes parâmetros foram analisados, tais como a velocidade de agitação (800 rpm, 1000 rpm e $1400 \mathrm{rpm}$ ), a fase externa ( $5 \mathrm{~mL}$ ou $20 \mathrm{~mL}$ ), as condições de $\mathrm{pH}$, o tipo de insulina, o tipo de surfactante (Pluronic ${ }^{\mathbb{R}} \mathrm{F} 68$ a $0,1 \%$ ou PVA a $2 \%$ ), as massas de PLGA (75 mg e $100 \mathrm{mg}$ ) e a presença/ausência de $\mathrm{NaCl}\left(0 \mathrm{~g} \mathrm{~L}^{-1}, 20 \mathrm{~g} \mathrm{~L}^{-1}, 50 \mathrm{~g} \mathrm{~L}^{-1}\right.$ ou $\left.100 \mathrm{~g} \mathrm{~L}^{-1}\right)$. As nanopartículas foram caracterizadas pelo seu tamanho e índice de polidispersividade (PDI) através do Delsa ${ }^{\mathrm{TM}}$ NanoC (Beckman Coulter, EUA). A morfologia foi observada por microscopia electrónica de varrimento (SEM). Uma alíquota de cada amostra, colocada numa lamela de vidro, foi deixada a secar num exsicador. $\mathrm{O}$ material foi em seguida revestido com uma fina camada de ouro (aproximadamente $500 \mathrm{~nm}$ de espessura) e observado num microscópio electrónico de varrimento JEOL 5200LV (JEOL Ltd., Tóquio, Japão), a uma voltagem de aceleração de $20 \mathrm{kV}$. As imagens foram adquiridas digitalmente. A Tabela 3 resume os diferentes parâmetros de formulações e de processos usados na preparação de todos os lotes produzidos $(\mathrm{A}-\mathrm{I})$.

\section{Eficiência de encapsulação (EE, \%)}

A percentagem de insulina encapsulada nas nanopartículas de PLGA foi determinada no sobrenadante (insulina não encapsulada após centrifugação a $14000 \mathrm{rpm}$, durante $10 \mathrm{~min}$ ) através de um método de HPLC isocrático, a $214 \mathrm{~nm}$, com detector UV, usando um fluxo constante de $0,8 \mathrm{~mL}$ min $^{-1}$ numa coluna LiChrospher 100, RP-18 $(5 \mu \mathrm{m}, 250 \mathrm{~mm} \times 4 \mathrm{~mm}$ i.d), da Merck, (Alemanha). Usou-se como fase móvel uma solução aquosa de $\mathrm{Na}_{2} \mathrm{SO}_{4}$ anidro $(0,2 \mathrm{M})$ ajustada a um $\mathrm{pH}$ de 2,3, com ácido fosfórico e acetonitrilo (de respectiva proporção em volume 73:27) [39]. Foi utilizada para avaliar a EE (\%) uma curva de calibração, com o limite 
mobile phase [39]. A calibration curve having linearity in the $1 \mathrm{ppm}$ to $100 \mathrm{ppm}$ interval was used to evaluate the EE (\%) by the formula: de linearidade entre 1 ppm e 100 ppm, a partir da seguinte fórmula: $\frac{\text { (Inicial insulin added }- \text { Insulin present in supernatant) }}{\text { Inicial insulin added }} \times 100$
(Insulina inicial adicionada - Insulina presente no sobrenadante) $X 100$ Insulina inicial adicionada

Tabela 3. Diferentes parâmetros de formulações e de processo usados.

Table 3. Different formulation and process parameters used.

\begin{tabular}{|c|c|c|c|c|c|}
\hline $\begin{array}{c}\text { Mass/ mass } \\
\text { PLGA, } m \text { / } \\
\text { mg }\end{array}$ & $\begin{array}{l}\text { Volume } \\
\text { External } \\
\text { phase/Fase } \\
\text { Externa, } v / \\
\text { mL }\end{array}$ & $\begin{array}{l}\text { Stirring speed/ } \\
\text { Velocidade de } \\
\text { agitação, } c \text { / } \\
\text { rpm }\end{array}$ & $\begin{array}{l}\text { Insulin/ } \\
\text { Insulina }\end{array}$ & $\begin{array}{l}\text { Surfactant/ } \\
\text { Surfactante }\end{array}$ & $\begin{array}{c}\text { Concentration } / \\
\text { Concentração } \\
\text { NaCl, } C \\
/ \%\end{array}$ \\
\hline $\begin{array}{ll}\text { A } 75 \\
\end{array}$ & 5 & 1400 & Actrapid $^{(B)}$ & F68 & 0 \\
\hline B 75 & 5 & 800 & Actrapid $^{\circledR}$ & F68 & 0 \\
\hline C 100 & 5 & 800 & Insuman $^{(B)}$ & PVA & 0 \\
\hline D 100 & 20 & 1000 & Insuman $^{(\mathbb{R}}$ & PVA & 5 \\
\hline E 100 & 20 & 1000 & Insuman $^{(1)}$ & F68 and PVA & 10 \\
\hline F 100 & 20 & 1000 & Insuman ${ }^{(\mathbb{1}}$ & PVA & 5 \\
\hline G 100 & 20 & 1000 & Novorapid $^{(\mathbb{R})}$ & F68 and PVA & 5 \\
\hline H 100 & 20 & 1000 & Novorapid $^{\mathbb{R}}$ & F68 and PVA & 2 \\
\hline I 100 & 20 & 1000 & Novorapid $^{(\mathbb{R})}$ & PVA & 10 \\
\hline
\end{tabular}

\section{Results and Discussion}

As seen in table 4, the multiple emulsion method seems to avoid the low affinity between the insulin (with a high MW and hydrophilic nature) and the PLGA. The presence of an internal reservoir inside the droplets in another phase may promote a major control of drug diffusion [40]. The PLGA concentration is an important factor that determines the reason between the drug/ polymer and consequently the EE [41]. On the other hand, the stirring speed controls the homogeneity and the particle size: in general the higher the agitation the smaller the particle size, but greater the difficulty in the recovery process. Higher speed rate can unbalance the osmotic equilibrium between the internal and the external phase and decreasing the EE [41]. Insulin can pass to the external phase or may also denature, thus affecting the drug stability [42]. With $1000 \mathrm{rpm}$, we got a more stable and homogeneous system. To maintain the integrity/stability of the emulsion, it is crucial the presence of surfactant agents. In this case, we used the F68 or the PVA only in the external phase or in the two phases. Factors like critical micelle concentration or the

\section{Resultados e Discussão}

De acordo com a tabela 4, o método de emulsão múltipla parece evitar a baixa afinidade entre a insulina, de elevado MM e natureza hidrofílica, com o PLGA. A presença de um reservatório interno nas gotículas de outra fase pode promover um maior controlo na difusão do fármaco [40]. A concentração do PLGA é um factor importante que determina a razão entre o fármaco/polímero e, consequentemente, a EE [41]. Por outro lado, a velocidade de agitação controla a homogeneidade e o tamanho de partícula, pois, em geral, quanto maior a agitação, mais pequeno será o tamanho da partícula produzida, mas maior a dificuldade no processo de isolamento. Além disso, uma elevada agitação pode interferir com o equilíbrio osmótico entre a fase interna e externa e diminuir a EE [41]. A insulina pode passar para a fase externa aquosa através de um processo de difusão ou mesmo poderá desnaturar, afectando assim a sua estabilidade [42]. A $1000 \mathrm{rpm}$, obtivemos um sistema mais estável e homogéneo. Para manter a integridade e estabilidade da emulsão é crucial a presença de agentes surfactantes. Neste caso, utilizámos o F68 ou o PVA, apenas na fase externa, ou ambos nas duas fases. Factores como a concentração micelar crítica ou o equilí- 
Hydrophilic-Lipophilic Balance (HBL) conditions, justified this choice. In the current study, PVA may be the most favorable surfactant. The higher value of insulin EE may also be supported by the presence of opposite charge between the species. Once the isoelectric point of insulin is around 5.3, positively charged insulin would interact strongly with negatively charged of the polymer $(\mathrm{pKa}=3)[43]$. At the same time, the protamine crystals in biphasic insulin may better control the release of insulin in formulation. A relevant aspect is the possible synergic interaction between those crystals and PLGA. Batches with Insuman showed the highest values of EE. The use of electrolytes in external phase decreased the particle size promoting the retention of insulin in the polymeric layers [44]. However, an excess of sodium salt can lead to an osmotic disequilibrium and consequently, to some particle destabilization. In fact, it was demonstrated that the $\mathrm{NaCl}$ concentrations of $20 \mathrm{~g} \mathrm{~L}^{-1}$ to $50 \mathrm{~g} \mathrm{~L}^{-1}$, not only decreased the particle size as well as increased the EE. In general, formulation D showed the best characteristics: lower size; lower polidispersivity index (PDI), that demonstrates the system homogeneity and a suitable EE. SEM observations revealed nanoparticles with a spherical shape that were not arranged in agglomerates (Fig. 2). brio hidrófilo-lipófilo (HBL) justificam a escolha destas condições. Neste estudo, o PVA mostrou ser o surfactante mais favorável. O elevado valor de EE da insulina poderá ser justificado pela presença de cargas opostas entre as espécies. Uma vez que o ponto isoelétrico (pI) da insulina é aproximadamente 5,3, a insulina carregada positivamente irá interagir fortemente com as cargas negativas do polímero $\left(\mathrm{pK}_{\mathrm{a}}=3\right)$ [43]. Simultaneamente, os cristais de protamina na insulina bifásica poderão controlar melhor a sua libertação da formulação. Um aspecto relevante é a possível interação sinérgica entre estes cristais e o PLGA. Os lotes com Insuman apresentaram valores mais altos de EE. O uso de electrólitos na fase externa diminuiu o tamanho da partícula promovendo a retenção da insulina nas camadas poliméricas [44]. No entanto, um excesso de cloreto de sódio poderá levar a um desequilíbrio osmótico e, consequentemente, à destabilização de alguma partícula. De facto, foi demonstrado que as concentrações de $\mathrm{NaCl}$ entre $20 \mathrm{~g}$ $\mathrm{L}^{-1}$ e $50 \mathrm{~g} \mathrm{~L}^{-1}$, não apenas diminuíram o tamanho da partícula como aumentaram a EE. Em geral, a formulação $\mathrm{D}$ revelou as melhores características: um baixo índice de polidispersividade (PDI) que demonstra a homogeneidade do sistema e uma EE adequada. As observações em SEM mostraram que as nanopartículas tinham uma forma esférica e não formavam aglomerados.

Os ensaios de HPLC mostraram a existência de um pico úni-

Table 4/ Tabela 4. Results of different formulation and process parameters Resultados dos diferentes parâmetros de formulação e do processo.

\begin{tabular}{cccc}
\hline $\begin{array}{c}\text { Batches } \\
\text { Lotes }\end{array}$ & $\begin{array}{c}\text { Mean size } \\
\text { Tamanho médio, } d / \mathrm{nm}\end{array}$ & PDI & EE / \% \\
\hline A & 1352.6 & 0.54 & 53.7 \\
B & 1598.9 & 0.36 & 48.8 \\
C & 1070 & 0.36 & 14.1 \\
D & 398.6 & 0.19 & 65.2 \\
E & 1283.8 & 0.007 & 60.4 \\
F & 631.3 & 0.27 & 60.6 \\
G & 942 & 0.31 & 50.6 \\
H & 760.4 & 0.34 & 56.0 \\
I & 2082.2 & 1.18 & 49.3 \\
\hline
\end{tabular}

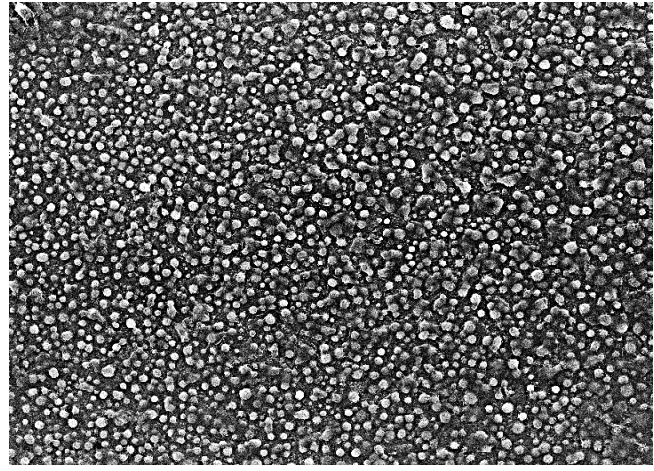

Figure 2/ Figura 2. SEM microphotograph of formulation D showing a large number of spherical nanoparticles. Scale bar $=100 \mu \mathrm{m}$.

Microfotografia em SEM da formulação D mostrando um grande número de nanopartículas esféricas. Escala $=100 \mu \mathrm{m}$.

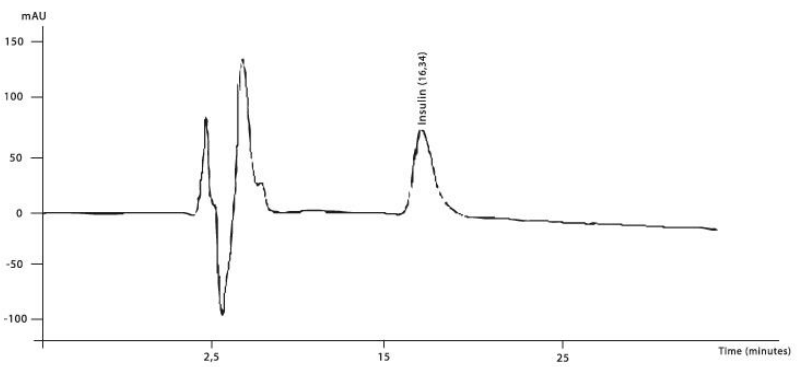

Figure 3/ Figure 3. Representative chromatogram of insulin (retention time at $16.34 \mathrm{~min}$ ).

Cromatograma representativo da insulina (tempo de retenção a $16,34 \mathrm{~min})$. 
HPLC assays showed a single and well-defined peak in the chromatogram (Fig. 3) and no degradation product was detected. The encapsulation process of insulin into PLGA nanoparticles maintained insulin retention time. The HPLC method was linear in the $1 \mathrm{ppm}$ to $100 \mathrm{ppm}$ concentration interval $\left(y=66.726 x\right.$, with $\left.\mathrm{R}^{2}=0.9995\right)$.

\section{Conclusions}

This study includes an initial phase of development and production of a new nanosystem as an alternative therapy in Diabetes Mellitus. Results suggest that insulin encapsulation into PLGA nanoparticles, produced by the previous methodology, has a great potential for oral delivery of this hormone. Coating of nanoparticles to counteract the future barriers along the GI tract is now necessary, as well as the in vitro and in vivo assays in order to demonstrate safety and effectiveness of this nanosystem.

\section{Conflict of Interests}

The authors declare that there are no financial and/or personal relationships that could be viewed as presenting a potential conflict of interests co bem definido no cromatograma (Fig. 3), não tendo sido detectado nenhum produto de degradação. O processo de encapsulação da insulina dentro das nanopartículas de PLGA manteve o tempo de retenção da insulina. O método de HPLC foi linear no limite de concentração entre 1 ppm e 100 ppm $\left(y=66,726 x\right.$, e $\left.R^{2}=0,9995\right)$.

\section{Conclusões}

Este estudo relata a fase inicial de desenvolvimento e produção de um novo nanossistema como alternativa terapêutica na Diabetes Mellitus. Os resultados sugerem que a encapsulação de insulina em nanopartículas de PLGA, produzidas por esta metodologia, parece ter potencialidades para a administração oral desta hormona. No entanto, o revestimento das nanopartículas, para contornar as possíveis barreiras ao longo do trato gastrointestinal terá de ser estudado, sendo necessários ensaios in vitro e in vivo que demonstrem a segurança e a eficácia deste nanossistema.

\section{Conflito de Interesses}

Os autores declaram não existir qualquer relação pessoal ou financeira que possa ser entendida como representando um potencial conflito de interesses. 


\section{Referências / References}

[1]. Ahmed I, el al. Diabetes mellitus. Clinics in Dermatology 2006; 24:237-46.

[2]. Dominguez LJ, et al. The discovery of insulin: what really happened 80 years ago. Annali Italiani di Medicina Interna 2001; 3:155-62.

[3]. Elliott JA, et al. Diabetes self-management and education of people living with diabetes: a survey in primary health care in muscat oman. PLoS One 2013; 2:1-7.

[4]. American Diabetes Association. Diagnosis and classification of diabetes mellitus. Diabetes Care 2010;33:S62-S69. in URL: http:// care.diabetesjournals.org.

[5]. Sharma N, et al. Oral Insulin - A Perspective Journal of Biomaterials Aplications 2003; 17:183-196.

[6]. American Diabetes Association. Standards of Medical Care in Diabetes -2013. Diabetes Care 2013;36:S11-S66 in URL: http://care. diabetesjournals.org

[7]. Blanco-Príeto F, et al. Polymeric particulates to improve oral bioavailability of peptide drugs. Molecules 2005; 10: 65-80.

[8]. Zhang L, et al. Improving the stability of insulin in solutions containing intestinal proteases in Vitro. International Journal of Molecular Sciences 2008; 9:2376-87.

[9]. Shive J, et al. Biodegradation and biocompatibility of PLA and PLGA microspheres. Advanced Drug Delivery Reviews 1997; 28:5-24.

[10]. Giuseppina Sandri M, et al. Insulin-loaded nanoparticles based on $\mathrm{N}$-trimethyl chitosan: In Vitro (Caco-2 model) and Ex Vivo (excised rat jejunum, duodenum, and ileum) evaluation of penetration enhancement properties. AAPS Pharmaceutical Science Technology $2010 ; 1: 362-71$.

[11]. Singh A, et al. Biodegradable nanoparticles are excellent vehicle for site directed in-vivo delivery of drugs and vaccines. Journal of Nanobiotechnology 2011; 9:1-11.

[12]. Shirui Mao O, et al. Uptake and transpor of PEG-graft-trimethyl-chitosan copolymerinsulin nanocomplexes by epithelial cells. Pharmaceutical Research 2005; 12:2058-68.

[13]. Mingguang Li Z, et al. Physiologically based pharmacokinetic modeling of PLGA nanoparticles with varied $\mathrm{mPEG}$ content. International Journal of Nanomedicine 2012; 7:1345-56.

[14]. Zhi Min Wua L, et al. HP55-coated capsule containing PLGA/RS nanoparticles for oral delivery of insulin. International Journal of Pharmaceutics 2012; 298:1-8.

[15]. Shive J, et al. Biodegradation and biocompatibility of PLA and PLGA microspheres. Advanced Drug Delivery Reviews 1997; 28:5-24.

[16]. Siegel H, et al. Poly Lactic-co-Glycolic Acid (PLGA) as biodegradable controlled drug delivery carrier. Polymers 2011; 3:1377-97.
[17]. Davaran S, et al. Adriamycin release from poly(lactide-coglycolide)-polyethylene glycol nanoparticles: synthesis, and in vitro characterization. International Journal of $\mathrm{Na}$ nomedicine 2006; 4:535-9.

[18]. Yin Q, et al. Targeting mantle cell lymphoma with Anti-SYK nanoparticles. Journal Annual Oncology. 2012; 1:1-9.

[19]. Sanchez H, et al. Cyclosporin A treatment increases rat soleus muscle oxidative capacities. Muscle Nerve 2003; 3:324-9.

[20]. Panyam J, et al. Rapid endo-lysosomal escape of poly(DL-lactide-co-glycolide) nanoparticles: implications for drug and gene delivery. FASEB Journal 2002; 10:1217-26.

[21]. Golla K, et al. A target-specific oral formulation of doxorubicin-protein nanoparticles: efficacy and safety in hepatocellular cancer. Journal Cancer 2013; 8:644-52.

[22]. Barichello JM, et al. Encapsulation of hydrophilic and lipophilic drugs in PLGA nanoparticles by the nanoprecipitation method. Drug Delivery Indian Pharmaceutical 1999; 7:221-34.

[23]. Martínez A, et al. Synthesis and characterization of alginate/chitosan nanoparticles as tamoxifen controlled delivery systems. Journal of Microencapsulation 2013; 4:398-408.

[24]. Kumar P, et al. Fluorescence behavior of non-functionalized carbon nanoparticles and their in vitro applications in imaging and cytotoxic analysis of cancer cells. Colloids Surface Biointerfaces 2012; 91:34-40.

[25]. Konan YN, et al. Preparation and characterization of sterile sub- $200 \mathrm{~nm}$ meso-tetra(4hydroxylphenyl)porphyrin-loaded nanoparticles for photodynamic therapy. Journal Pharmaceutical Biopharmacology 2003; 1:115-24

[26]. Nair HB, et al. Delivery of antiinflammatory nutraceuticals by nanoparticles for the prevention and treatment of cancer. Biochemistry Pharmacology 2010; 12:1833-43.

[27]. Koppolu B, et al. Development of multiplelayer polymeric particles for targeted and controlled drug delivery. Nanomedicine 2010; 2:355-61.

[28]. Saeki D, et al. Microfluidic preparation of water-in-oil-in-water emulsions with an ultra-thin oil phase layer. Lab on a Chip 2010; 3:357-62.

[29]. Kashi TS, et al. Improved drug loading and antibacterial activity of minocycline-loaded PLGA nanoparticles prepared by solid/oil/ water ion pairing method. International Journal of Nanomedicine 2012; 7:221-34

[30]. Sun S, et al. Insulin-S.O (sodium oleate) complex-loaded PLGA nanoparticles: formulation, characterization and in vivo evaluation. Journal of Microencapsulation 2010; 6:471-8.
[31]. Sun S, et al. Hydrophobic ion pairing of an insulin-sodium deoxycholate complex for oral delivery of insulin. International Journal of Nanomedicine 2011; 6:3049-56

[32]. Qian F, et al. Chitosan graft copolymer nanoparticles for oral protein drug delivery: preparation and characterization. Biomacromolecules 2006; 10:2722-7.

[33]. Reis CP, et al. Nanoparticulate delivery system for insulin: design, characterization and in vitro/in vivo bioactivity. European Journal of Pharmaceutical Sciences 2007; 5:392-7.

[34]. Morales-Cruz M, et al. Two-step nanoprecipitation for the production of protein-loaded PLGA nanospheres. Results in Pharmaceutical Sciences 2012; 2:79-85.

[35]. Singnurkar PS, et al. Evaluation of hydrophobic nanoparticulate delivery system for insulin. Indian Journal of Pharmaceutical Sciences 2008; 6:721-6.

[36]. Jain S, et al. Folate-decorated PLGA nanoparticles as a rationally designed vehicle for the oral delivery of insulin. Nanomedicine 2012; 9:1311-37.

[37]. Li X, et al. Nanoemulsions coated with alginate/chitosan as oral insulin delivery systems: preparation, characterization, and hypoglycemic effect in rats. International Journal of Nanomedicine 2013; 8:23-3.

[38]. Jiau J, et al. Rheology and stability of waterin-oil-in-water multiple emulsions containing Span 83 and Tween 80. AAPS Pharmaceutical Sciences 2003; 1:7.

[39]. Xu X, et al. Quantitative determination of insulin entrapment efficiency in triblock copolymeric nanoparticles by high performance liquid chromatography. Journal of Pharmaceutical and Biomedical Analyses 2006; 1:266-73.

[40]. Ilek N, et al. A lecithin-based microemulsion of rh-insulin with aprotinin for oral administration: Investigation of hypoglycemic effects in non-diabetic and STZ-induced diabetic rats. International Journal of Pharmaceutics 2005; 298:176-185.

[41]. Gaudana R, et al. Encapsulation of ProteinPolysaccharide HIP Complex in Polymeric Nanoparticles. Journal Drug Delivery 2011; 1-7.

[42]. Moraima Morales-Cruz G, et al. Two-step nanoprecipitation for the production of protein-loaded PLGA nanospheres. Results Pharmaceutical Sciences 2012; 2:79-85.

[43]. Sarmento B, et al. Oral insulin delivery by means of solid lipid nanoparticles. International Journal of Nanomedicine 2007; 4:743749

[44]. Yeh M, et al. In vivo and in vitro characteristics for insulin-loaded PLA microparticles prepared by w/o/w solvent evaporation method with electrolytes in the continuous phase. Journal of Microencapsulation 2004; 7:719-728. 